\title{
Mal-adaptation of event-related EEG responses preceding performance errors
}

\author{
Heike Eichele ${ }^{1}$, Hilde T. Juvodden ${ }^{1}$, Markus UIlsperger ${ }^{2,3}$ and Tom Eichele ${ }^{1,4}$ * \\ Department of Biological and Medical Psychology, University of Bergen, Bergen, Norway \\ 2 Max Planck Institute for Neurological Research, Cologne, Germany \\ ${ }^{3}$ Donders Institute for Brain, Cognition and Behaviour, Radboud University Nijmegen, Netherlands \\ ${ }^{4}$ Mind Research Network, Albuquerque, NM, USA
}

Edited by:

MichaelX. Cohen, University of

Amsterdam, Netherlands

Reviewed by:

James F. Cavanagh, Université Paris

Descartes, France

Ali Mazaheri, University of California

at Davis, USA

${ }^{*}$ Correspondence:

Tom Eichele, Department of Biological and Medical Psychology, University of Bergen, Jonas Lies Vei 91, 5009

Bergen, Norway.

e-mail: tom.eichele@psybp.uib.no
Recent EEG and fMRI evidence suggests that behavioral errors are foreshadowed by systematic changes in brain activity preceding the outcome by seconds. In order to further characterize this type of error precursor activity, we investigated single-trial event-related EEG activity from 70 participants performing a modified Eriksen flanker task, in particular focusing on the trial-bytrial dynamics of a fronto-central independent component that previously has been associated with error and feedback processing. The stimulus-locked peaks in the N2 and P3 latency range in the event-related averages showed expected compatibility and error-related modulations. In addition, a small pre-stimulus negative slow wave was present at erroneous trials. Significant error-preceding activity was found in local stimulus sequences with decreased conflict in the form of less negativity at the N2 latency $(310-350 \mathrm{~ms})$ accumulating across five trials before errors; concomitantly response times were speeding across trials. These results illustrate that error-preceding activity in event-related EEG is associated with the performance monitoring system and we conclude that the dynamics of performance monitoring contribute to the generation of error-prone states in addition to the more remote and indirect effects in ongoing activity such as posterior alpha power in EEG and default mode drifts in fMRI.

Keywords: performance monitoring, errors, event-related EEG, independent component analysis, single trial analysis, deconvolution

\section{INTRODUCTION}

"It is hardly surprising to find that the organism's response to "identical" stimuli is in flux. The nervous system is not a passive recipient of inputs that are obediently switched to outputs; rather it is a dynamic system that continuously generates hypotheses about the environment"

(Squires et al., 1976).

It is trivial to state that human behavior and human brain activity are highly variable, yet more than thirty years after presenting the astonishing EEG data that led Squires and colleagues to write these lines, echoing James' prescient perception (James, 1890, the digitized text is freely available at http://www.archive.org/) the core assumption in cognitive neuroscience and its neuroimaging methods still maintains that there is a deterministic event-related signal and random noise. This assumption is used to justify averaging the rich and complex information in EEG and fMRI measurements for denoising and data reduction (in event-related EEG simply by taking the mean across trials, in fMRI through first-level modeling with fixed predictors). This happens, we assume, for analytical convenience and traditional reasons, although the average signal does not at all reflect a great deal of variability in the raw data (roughly about $10 \%$ ), and is not necessarily representative of the single trials at which some behavior occurred (Arieli et al., 1996; Raichle and Snyder, 2007). The challenge for cognitive neuroscience in a time when processors are fast and memory is cheap is to utilize the many elegant methods for single-trial analysis that are (mostly freely) available and peek into the exciting dynamics of brain activity on a moment-to-moment basis. In order to make the case, we present an example from event-related EEG where a combination of data decomposition with independent component analysis (ICA), multiple regression and deconvolution is used to derive a heretofore unknown electrophysiological precursor of behavioral errors.

Since the observation of the medial frontal negativity in the response-locked event-related potential associated with error commission (Falkenstein et al., 1991; Gehring et al., 1993), brain activity patterns and behavioral changes that are caused by errors have received a great deal of attention and helped in understanding how the brain responds to errors, and shapes subsequent behavioral adaptation. A neural system located mainly within the rostral cingulate zone (RCZ), the pre-supplementary motor area (pre-SMA) and the anterior insular cortex has been identified to support this function by signaling the need for increased control, whenever the action goal is not achieved or the risk to fail is high (Ridderinkhof et al., 2004; Debener et al., 2005; Klein et al., 2007).

In contradistinction, changes in brain activity and behavior preceding errors have received much less attention, although these antecedent conditions may help to understand how brain states affect behavioral accuracy, and activity patterns preceding errors might lend themselves to prediction of upcoming performance, which would possibly have a number of interesting real-world applications. To date, only a limited amount of studies have investigated EEG activity immediately preceding errors; these have employed different EEG features for analysis and the evidence points to 
different, but quite possibly interrelated sources of performance errors. Briefly summarized, in trials preceding errors event-related EEG shows more positive response-locked activity (Ridderinkhof et al., 2003; Allain et al., 2004; Hajcak et al., 2005), reduced theta power (Cavanagh et al., 2009), decreased amplitudes of stimuluspreceding contingent negative variation and the stimulus-following P300 components (O'Connell et al., 2009), while $\alpha$ and $\mu$ rhythm power increase (Mazaheri et al., 2009).

In addition, changes expanding on a longer timescale across trials before errors can be observed in behavioral, electrophysiological, and hemodynamic measures: responses are executed increasingly more quickly (Smith and Brewer, 1995; Gehring and Fencsik, 2001), frontal and insular regions associated with attention regulation and effort show a graded decline of hemodynamic activation (Eichele et al., 2008), while event-related responses in regions within the default mode network (Raichle et al., 2001) show reduced deactivation (Li et al., 2007; Eichele et al., 2008). Similarly, an increase in right parietally localized $\alpha$-power in the scalp EEG evolves across the same timescale (O'Connell et al., 2009).

We have previously suggested that such trends in the trial-bytrial dynamics of hemodynamic activity preceding errors stem from a (mal-)adaptive system rather than spontaneous variability. As such, we posit that error-preceding activity more or less directly represents parts of the systems that mediate error monitoring and more general cognitive control functions (Ridderinkhof et al., 2004; Ullsperger and von Cramon, 2004). In the context of cognitive control models (Yeung et al., 2004; Yu et al., 2009) this account predicts that variations of the stimulus sequence that impact on conflict (and attention) are continuously employed to adapt the system, and thus are reflected in the dynamics of event-related responses generated by the system. We imply more generally that a representation of predictive information transferred in the stimulus history is continuously utilized to optimize brain responses (Sutton et al., 1965; Squires et al., 1976; Eichele et al., 2005; Friston, 2005; Raichle, 2006; Botvinick, 2007; Mars et al., 2008). This optimization yields adjustments of overt response speed and commensurate corticospinal excitability when events are repetitive and subjectively predictable (Huettel et al., 2002, 2005; Bestmann et al., 2008) and consequently affect the trade-off between speed and accuracy (Forstmann et al., 2008; Ivanoff et al., 2008; van Veen et al., 2008).

Here, we employed a modified Eriksen flanker task (Eriksen and Eriksen, 1974; Gratton et al., 1992) and the event-related responses in this task are modulated by conflict in particular during the latency of the $\mathrm{N} 2$ from about $250 \mathrm{~ms}$ after stimulus onset, extending well into the latency of the P3 up to around $500 \mathrm{~ms}$ (Kopp et al., 1996; Yeung et al., 2004; Folstein and Van Petten, 2008). Errors occur relatively more often to incompatible trials, in particular when these follow low conflict compatible trials and yield a large error-related negativity (ERN) in the response-locked EEG at about $80 \mathrm{~ms}$ with a subsequent positivity (Falkenstein et al., 2000; Debener et al., 2005).

The present study focuses on the dynamics of a medial frontal EEG source with a generator in the RCZ that previously has been associated with error and feedback processing (Roger et al., 2010; Debener et al., 2005; Gentsch et al., 2009), providing the scalp electrophysiological surrogate for the performance monitoring system. We aimed to show that this source reflects mal-adaptive event- related activity preceding erroneous performance at the time of conflict/compatibility processing, in particular we expect gradually lower amplitudes of event-related responses similar to our previous fMRI findings. This finding would link error precursors more directly to performance monitoring, rather than to unspecific ongoing activity producing spurious noise signals that lead to random lapses in performance.

In order to separate the medial frontal source from other eventrelated responses and background activity we employed temporal ICA (Bell and Sejnowski, 1995) and identified for each participant the source that jointly topographically and functionally reflected conflict and error responses (IE) and then analyzed the trial-by-trial by dynamics surrounding errors. Due to its role in (pre-response) conflict processing and temporal overlap with the ERN we expected in particular the $\mathrm{N} 2$ amplitude to show a gradually lesser negativity prior to errors. However, we inspected the entire time period surrounding stimulus onset to pick up trends localized to preceding responses (Ridderinkhof et al., 2003), as well as stimulus-preceding negativities and P3 (O'Connell et al., 2009).

\section{MATERIALS AND METHODS PARTICIPANTS}

Seventy participants ( 29 male, 41 female) are included in this study and were recruited from psychology and medicine undergraduate classes at the University of Bergen. Written informed consent was obtained from all participants and the study was approved by Regional Committee for Medical Research Ethics, West-Norway. Included participants had normal, or corrected to normal vision, and no history of neurological or psychiatric disorders and present use of psychotropic medication. Eight of the participants were left-handed individuals. The mean age of the participants was 22.02 years $( \pm 3.01)$. Data from five additional participants were discarded due to excessive non-stereotyped EEG artifacts.

\section{EXPERIMENTAL DESIGN}

After verbal and written instruction and a training sequence, participants performed a modified visual Eriksen flanker task implemented in E-prime 2 (Psychology software tools). At the center of a PC screen, participants were presented a fixation dot. Trials began with the presentation of six horizontal flanker-arrows appearing below the fixation. Participants were instructed to respond as fast as possible and as accurate as possible with either a left or a right mouse button press following the direction of a central target arrow that appeared $100 \mathrm{~ms}$ after the flankers. The central target arrow pointed either into the same direction as the flanker-arrows in compatible trials $(<<<<<<<,>>>>>>>)$ or in the opposite direction in incompatible trials $(<<<><<<,>>><>>>)$. The target and flankerarrows remained on screen until a response was registered. Trials were terminated by the motor response and were followed by a fixed $800 \mathrm{~ms}$ interval before the onset of the next trial. Stimuli were presented in five blocks with 200 trials that were pseudo-randomized separately for each participant. No performance feedback was given during the experiment. The overall probability of compatible and incompatible trials, as well as left and right responses was kept at 0.5 , respectively. Local probability manipulations of compatibility, response and stimulus-type were embedded in the stimulus sequences: twenty four participants received stimulus sequences in 
which compatibility was parametrically varied in short sequences of 40 trials in which the ratio between the compatible and incompatible trials was $1 / 9,3 / 7,5 / 5,7 / 3$ or $9 / 1$, respectively, while the probability of a left/right response was kept at 0.5 . Twenty three participants received stimulus sequences in which response side was similarly varied in sequences of 40 trials with ratios between left and right of $1 / 9,3 / 7,5 / 5,7 / 3$ or $9 / 1$, respectively, while conflict was kept at 0.5 . Twenty three participant received sequences with sequences of 50 trials in which one of the trial type appeared at $p=0.7$, and the other three stimuli at $p=0.1$ respectively. Here, we only focus on error-preceding activity which is present in all three experiments, and will report the main effects of parametric manipulations elsewhere.

\section{TRIAL SEOUENCE}

Errors in the flanker task are not randomly distributed but occur more frequently at high conflict incompatible trials. In order to visualize and analyze the relationship between errors and the preceding stimulus sequence the compatibility was coded in a binary vector containing all trials, where -1 denoted compatible, and +1 denoted incompatible trials, respectively. These were then used to derive the average occurrences from five trials prior to five trials after errors (see Figure 4, top panel).

\section{BEHAVIORAL ANALYSIS}

Response time (RT) and response accuracy (RACC) averages were generated for all possible outcomes. Responses faster than $100 \mathrm{~ms}$ $(0.03 \%)$ and slower than $1000 \mathrm{~ms}(0.48 \%)$ were not considered. Errors were defined as incorrect key presses to compatible and incompatible trials. The categorical effects of compatibility, stimulus/response repetition and accuracy were removed from the singletrial RTs by means of multiple linear regression (Notebaert and Verguts, 2007). The RACC data for each participant were coded as a binary vector with 0 representing correct outcomes, and 1 errors, and were then used to derive the residual modulation from five trials prior to five trials after error commission using the deconvolution method we have introduced for hemodynamic response estimation previously (Eichele et al., 2008). In brief, we take the Moore-Penrose pseudo-inverse of the convolution matrix containing the $-5 \ldots+5$ lagged versions of the accuracy predictor and multiply this with the RT/EEG vector yielding the residual modulation surrounding the error as the output. An illustration of the method with a simulation example is provided in Figure 1, for further details and areas of application see our previous work (Eichele et al., 2008, 2009).

Deconvolution enables inclusion of overlapping trial sequences with errors and is preferable to within-subject averaging here. The deconvolved RT modulation was tested with point-wise one-sample
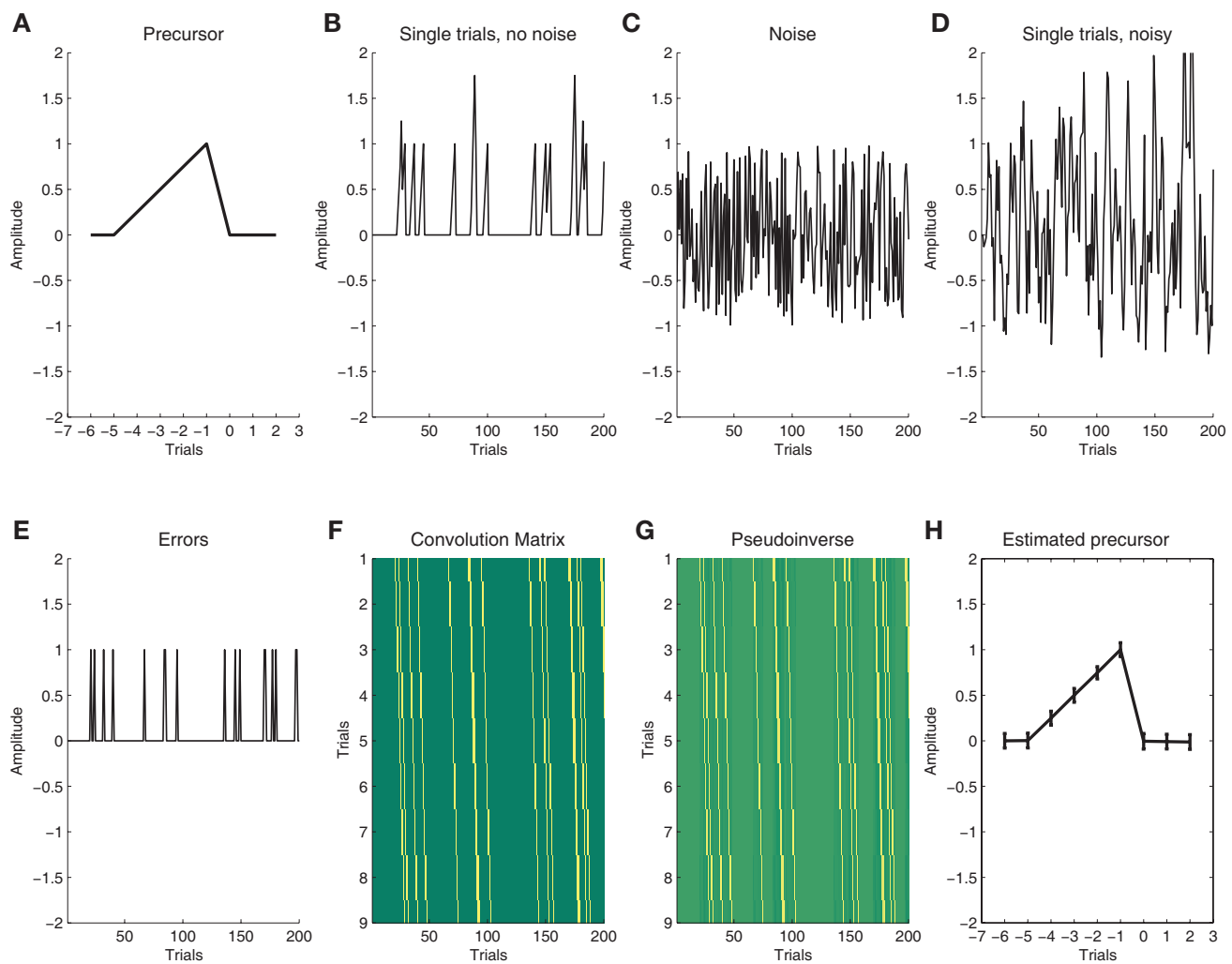

FIGURE 1 | Illustration of deconvolution of latent sequential effects. (A) We assume a latent precursor signal that gradually evolves across trials and precedes each error; (B) In order to illustrate a noiseless sequence of single trials we convolve a vector with $10 \%$ errors at random instances, note the summation of overlapping sequences; (C) for real data, we assume additional noise; (D) Convolution of the noisy data $(\mathbf{C}+\mathbf{E})$ with $(\mathbf{A})$ yields the simulated single-trial data that are used for deconvolution; (E) In order to deconvolve the data, we use a "Stick"-function that describes the occurrence of errors; (F) stacking the stick function at different lags is the convolution matrix; (G) we then take the pseudo-inverse of (F) and multiply the resulting matrix with the noisy data (D); (H) The product is an estimate of the precursor. In this figure, we show the average across 100 runs, error bars indicate the \pm 1 standard deviation around the mean. 
$t$-tests against zero mean; in addition a linear slope was fitted to the five error-preceding trials using linear regression. The resulting scaling factors were also subjected to a one-sample $t$-test to provide "random" effects population inferences (Figure 4, middle panel).

\section{EEG ACOUISITION}

EEGs were recorded continuously inside an electromagnetically and acoustically shielded chamber (Rainford EMC Systems, Wigan, UK) at $1-\mathrm{kHz}$ sampling frequency (low cutoff at $0.1 \mathrm{~Hz}$ and a high cutoff at $250 \mathrm{~Hz}$ ) with BrainAmp MR plus X2 amplifiers (BrainProducts, Munich, Germany). Participants were fitted with an elastic cap (Braincap, FMS, Falk Minow Services, Herrsching, Germany) containing $61 \mathrm{Ag} / \mathrm{AgCl}$ electrodes placed at Fp1, Fpz, Fp2, AF7, AF3, AFz, AF4, AF8, F7, F5, F3, F1, Fz, F2, F4, F6, F8, FT7, FC5, FC3, FC1, FCz, FC2, FC4, FC6, FT8, T7, C5, C3, C1, Cz, C2, C4, C6, T8, TP7, CP5, CP3, CP1, CPz, CP2, CP4, CP6, TP8, P7, P5, P3, P1, Pz, P2, P4, P6, P8, PO7, PO3, POz, PO4, PO8, O1, Oz, O2, TP9 and TP10. Vertical eye movements were monitored with a bipolar derivation between Fp1 and an additional electrode placed below the left eye. Additionally, ECG was monitored. Channels were referenced to TP9 with a ground on the right cheek and impedances were kept below $10 \mathrm{k} \Omega$.

\section{EEG PROCESSING}

The EEG data were offline re-referenced to common average reference, filtered from 0.5 to $45 \mathrm{~Hz}(12 \mathrm{db})$, and decimated to $500 \mathrm{~Hz}$ sampling rate. The data were then divided into epochs spanning from before the preceding response $(-900 \mathrm{~ms}$ relative to target onset) to after the current response (+1100 ms after target onset), the mean value of the entire epoch served as baseline. Segments containing large, non-stereotyped artifacts with amplitudes exceeding $\pm 300 \mu \mathrm{V}$ on any of the channels were rejected, and padded with the average of adjacent trials. Hereafter, the 61 scalp channels from each dataset were subjected to temporal ICA using infomax (Bell and Sejnowski, 1995), implemented in EEGLAB (Delorme and Makeig, 2004), estimated 30 components after PCA compression. For an overview about ICA and its applications to neuroimaging data see (Makeig et al., 2004; Stone, 2004; Calhoun et al., 2009; Eichele et al., 2009). In order to cluster components for further analysis and identify the most relevant to conflict and error processing we used automated sorting routines. Firstly, correlation with spatial templates for blink and lateral eyes movements was used to identify ocular artifacts (Viola et al., 2009). Secondly, muscular and other artifacts mainly localized to single electrodes were identified through the spatial standard deviation of the topography and correlation with a spectral template. From the remaining components, one was extracted for each participant that best matched the expected fronto-central topography, with large amplitude errorrelated activity and a conditional difference between compatible and incompatible trials (Roger et al., 2010; Debener et al., 2005; Gentsch et al., 2009). In most participants this procedure yielded a single matching component. In one participant the topography did not match the spatial template. In about $10 \%$ of the sample, two or three components matched the criteria, and we selected the one with the largest correlation score. Topographies of the selected components were assigned a positive maximum, and the component activations were then back projected onto sensor level to recover the sign and amplitude of the scalp recorded potentials.

\section{INFERENCE}

In order to test the consistency of topographies across participants, the channel weights were entered into one-sample $t$-tests under the assumption of zero magnitude. For visualization, topographies were also averaged, and a random sample of six individual topographies is provided in Figure 2.

Amplitude differences of individual component averages for compatible and incompatible correct (IC) trials were tested with paired $t$-tests at all time points to derive the latency and magnitude of the conflict effect; similarly, the difference between component averages for IC trials and error trials were subjected to paired $t$-tests to test for error-related responses.

In order to estimate error-preceding activity not predicted by the categorical effects of compatibility, stimulus/response repetition and accuracy, which themselves exert significant influences on the event-related response we removed the variability associated with these predictors from the single-trial EEG at each time point by means of multiple linear regression. In effect, the N2-P3 amplitude difference due to conflict, the error-specific response (ERN/Pe), and unspecific repetition priming (Mayr et al., 2003; Ullsperger et al., 2005) were subtracted from the data before further analysis. As for the behavioral data, the RACC vectors were used to deconvolve the residual event-related EEG modulation from five trials prior to five trials after error commission for the average amplitude in the latency window around the maximal N2 conflict effect (310-350 ms). The residual event-related modulation was tested with point-wise one-sample $t$-tests against zero mean; in addition a linear gradient was fitted to the five error-preceding trials using linear regression. The resulting scaling factors were also subjected to a one-sample $t$-test. Additional latency windows around stimulus onset, the early sensory evoked responses, P3 and post-response slow waves were also explored but yielded no significant residual effects preceding errors (data not shown).

Effects of all statistical tests were significant at an uncorrected $t$-threshold of $p<0.002$ for two-tailed tests.

\section{RESULTS STIMULUS SEQUENCE}

Eighty two percent of all errors occurred to incompatible trials, an expected outcome in the flanker task. Error-preceding trials showed an increased frequency of compatible trials (63\%), the linear fit across five preceding trials resulted in an average $2 \%$ per trial increase of compatible trials $\left(t_{69}=-6.27\right)$. Following erroneous responses, the probability of compatible/incompatible outcomes expectedly returns to 0.5 . Figure 4 (top panel) shows the modulation of compatibility surrounding error trials.

\section{BEHAVIOR}

The RT and RACC results yielded a pattern typical for the Flanker task. Compatible correct (CC) responses were associated with fast RT (362 ms \pm 0.62$)$ and low error rate $(2.0 \% \pm 0.02)$, while incompatible trials showed slower RT $(461 \mathrm{~ms} \pm 0.72)$ and more frequent errors $(9.2 \% \pm 0.08)$ with fast RT (309 ms \pm 0.89$)$. Residual RTs were on average $11 \mathrm{~ms}( \pm 2.42)$, faster in trials immediately prior to errors $\left(t_{69}=-4.76\right)$. Across the five error-preceding trials, the linear fit indicated average speeding by $2.5 \mathrm{~ms}( \pm 0.56)$ per trial $\left(t_{69}=-4.51\right)$. After error trials post-error slowing of responses by $42 \mathrm{~ms}( \pm 6.15)$ was 

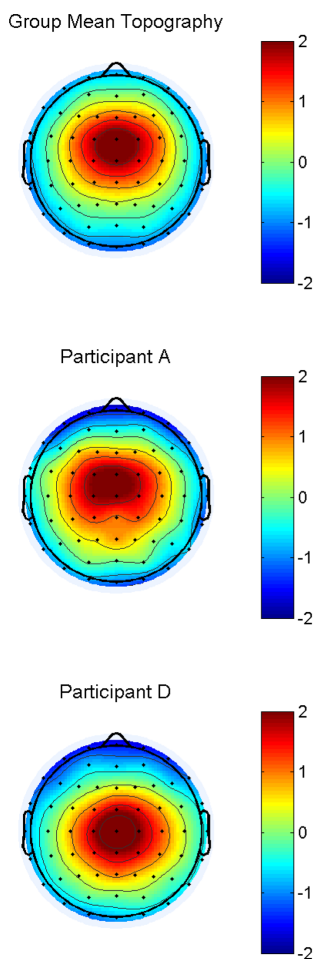
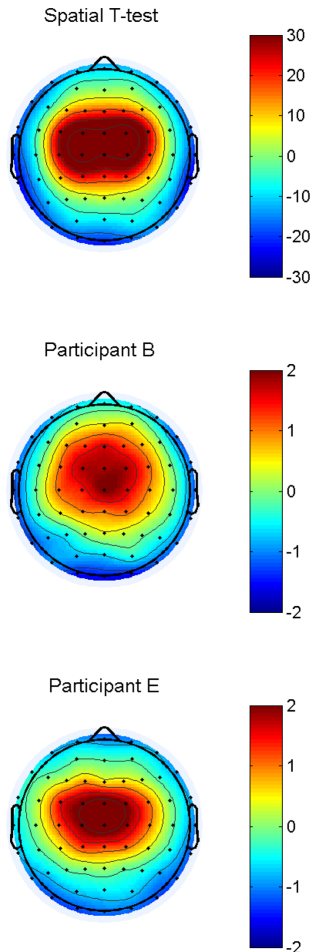
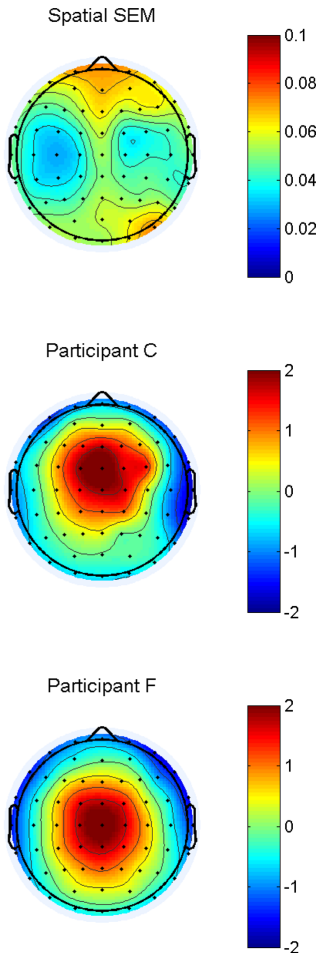

FIGURE 2 |Top row, left: the group average topography, scaled from -2 to $+2 \mu \mathrm{V}$ shows a clear maximum at $\mathrm{FC} z$, extending to the neighboring electrodes FC, FC2, C1, Cz and C2. The spatial T-map (top row, middle) and the corresponding map of the standard error of the mean (SEM) illustrate the robustness of the selected topography in the sample. The middle and bottom rows (participants A-F) provide six randomly drawn single subject replications. observed $\left(t_{69}=6.87\right)$. In subsequent trials sustained slowing on the order of 15 ms was present (all trials $p<0.001$ ). The RT modulation around errors is shown in Figure 4 in the middle panel.

\section{EEG}

The component topographies were fairly stable across participants, with an average maximum at $\mathrm{FCz}\left(2.31 \pm 0.06, t_{69}=40.97\right)$; the average of the spatial correlation between all individual topographies was 0.83 .

In the component time courses the largest differences between CC and IC responses were seen during the latency of the N2 at $316 \mathrm{~ms}$ post stimulus with a $0.95 \mu \mathrm{V}$ more negative peak in incompatible trials $\left(t_{69}=-7.12\right)$; at $430 \mathrm{~ms}$ the $\mathrm{P} 3$ peak was $1.21 \mu \mathrm{V}$ larger $\left(t_{69}=6.96\right)$. Error trials yielded a large negativity at $350 \mathrm{~ms}$ post stimulus $\left(-3.76 \mu \mathrm{V}, t_{69}=-12.01\right)$, corresponding to the responselocked ERN, and a subsequent positivity at $546 \mathrm{~ms}(3.54 \mu \mathrm{V}$, $\left.t_{69}=12.71\right)$. In addition, we observed a small negativity $(-0.40 \mu \mathrm{V}$, $\left.t_{69}=-5.29\right)$ that preceded stimulus onsets with subsequent IE by approximately 200-300 ms, and which sustained through the early $\mathrm{P} 1$ and $\mathrm{N} 1$ peaks of the component average.

These effects are visible in the independent component event-related potentials (ICERPs) in the bottom sections of Figure 3, to the left separately for CC, IC, and IE, to the right as difference waves.

In addition to ICERPs we visualized the dependency of the stimulus-locked response on RT by sorting the epochs by RT (Figure 3, top left, gray line represents the average sorted RT), and averaging the resulting single-trial image across participants. For orientation, we also overlaid the relative frequency of incompatible trials (blue line), and errors (red line). The figure illustrates in particular $\mathrm{N} 2$ and $\mathrm{P} 3$ preceding the response in mainly correct trials with slower RT (top half of the image), and ERN and PE following the response at error trials (bottom quarter of the image). Correspondingly, the top right section of the figure presents the average RACC across the participants when the data are sorted by the component amplitude at each time point and shows higher than mean error rates in red, and lower than mean error rates in blue. The dominant feature in the figure is the scaling of error rate with increasing negative amplitudes during the N2/ERN latency and during the pre-stimulus negativity, and inversely during the $\mathrm{P} 3 / \mathrm{Pe}$ latency range.

The residual amplitude at the $\mathrm{N} 2$ latency was on average $0.34 \mu \mathrm{V}( \pm 0.08)$ more positive in trials immediately prior to errors $\left(t_{69}=4.11\right)$. Correspondingly, a positive-going trend of $0.07 \mu \mathrm{V}( \pm 0.02)$ was estimated across the five error-preceding trials $\left(t_{69}=2.99\right)$. Following errors, N2 estimates returned to baseline. The EEG modulation around errors is shown in Figure 4 in the bottom panel.

\section{DISCUSSION}

This study addressed the question whether errors are associated with antecedent (mal-)adaptation of the performance monitoring system effective during conflict processing indexed by the N2 amplitude modulation in a medial frontal independent source (Roger et al., 2010; Debener et al., 2005; Gentsch et al., 2009). 

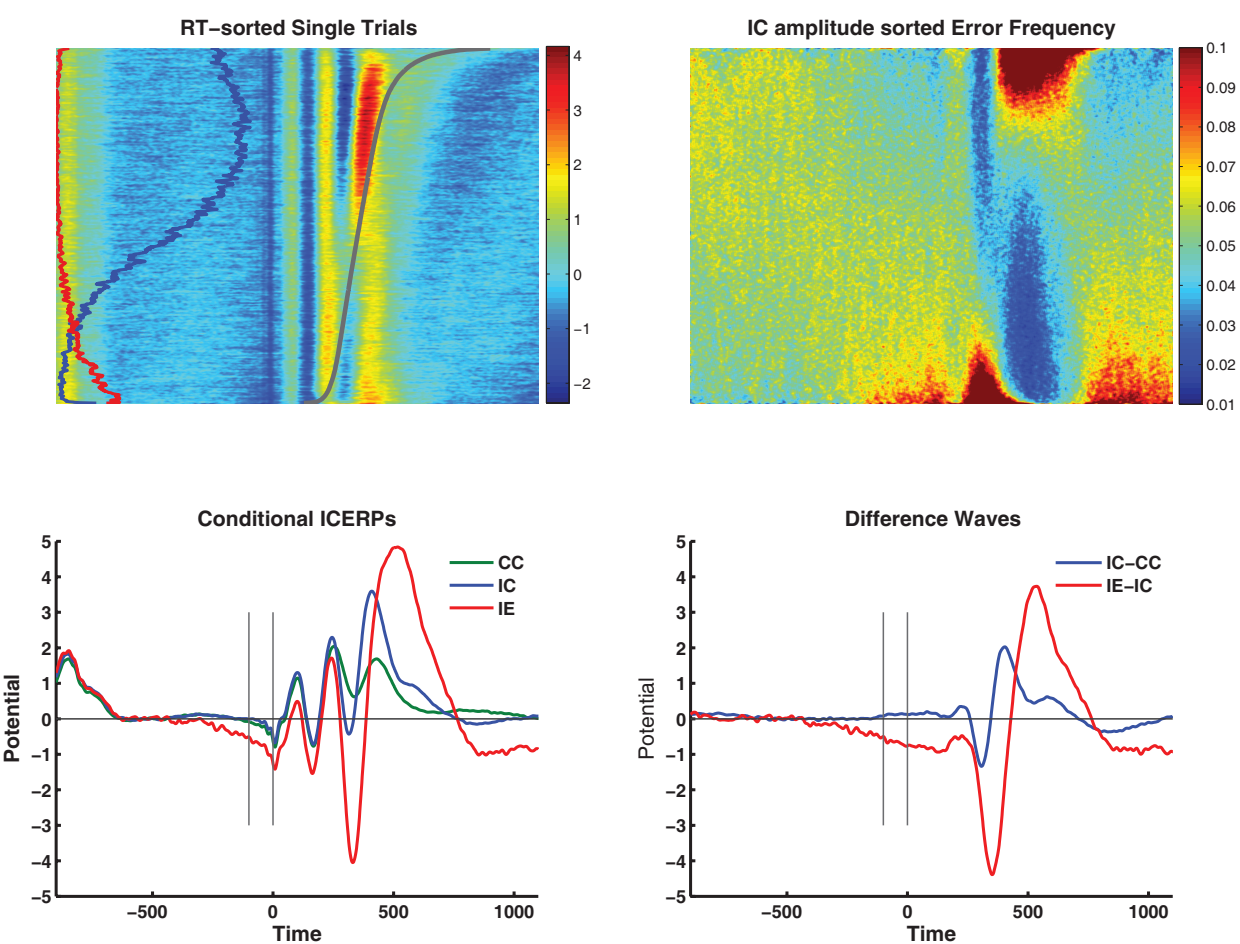

FIGURE 3 |Top left: Group average RT-sorted single-trial ICERP image showing the dependency of the stimulus-locked response on RT (gray line). The relative frequency of incompatible trials is given on the left of the image in blue, the proportion of errors in red. The figure illustrates in particular $\mathrm{N} 2$ and P3 preceding the response in mainly correct trials with slower RT (top half of the image), and ERN and PE following the response at error trials (bottom quarter of the image). Top right: Shows the average accuracy across the group sorted by the component amplitude at each time point and shows higher error

rates in red, and lower than error rates in blue (equivalent to "vincentizing"). The dominant feature in the figure is the scaling of error rate with increasing negative amplitudes during the N2/ERN latency and during the pre-stimulus negativity, and inversely during the P3/Pe latency range. Bottom left: conditional ICERPs for compatible correct (CC, green), incompatible correct (IC, blue), and error responses (IE, red). Bottom right: difference waves for incompatible correct minus compatible correct (blue) and incompatible errors minus incompatible correct (red).

In order to unmix the scalp EEG correlates of performance monitoring from unrelated event-related responses and background rhythms we employed blind source separation with infomax ICA in the time domain and used correlation-based clustering of individual component topographies and time courses to identify this source across a large sample of individual datasets. From inspection and statistical analysis of the data presented in Figures 1 and 2 we assume that the decomposition allows focusing selectively on the best surrogate for performance monitoring and affords a singletrial analysis of this source without latent confounds from other concurrent processes (Makeig et al., 2004; Onton et al., 2006), thus the error-preceding activity changes reported here should be largely unrelated to lapses that relate to spontaneous fluctuations of intrinsic activity.

The ICERPs showed a typical pattern of results with an N2-P3 conflict effect (Forster et al., 2010; Folstein and Van Petten, 2008) and a large (albeit stimulus-locked) ERN/Pe to error trials (Falkenstein et al., 1991; Gehring et al., 1993). Of note, erroneous trials showed a sustained negativity that significantly deviated from baseline already before flanker/target onset (Figure 3, bottom left), suggesting that (premature) error commission indeed starts before the stimulus has arrived. In terms of topography and timing this negativity may be related to preparatory potentials such as the (typically much larger) contingent negative variation that have sources in the medial walls of the frontal lobes (Nagai et al., 2004). Alternatively, this deflection may represent asymmetric oscillations around stimulus onset (Mazaheri and Jensen, 2008), possibly in the theta-range which also has been associated with this topography during task processing (Onton et al., 2005) and relaxed resting (Scheeringa et al., 2008). Although a long trend across trials was not present this negativity deserves further study.

The results shown in Figure 4 yield three error-preceding phenomena. First: an increasing frequency of compatible trials prior to mainly incompatible errors. Second: speeding of RTs similar to previous reports (Smith and Brewer, 1995; Gehring and Fencsik, 2001). Third: a positive-going trend in the N2 latency range of the IC event-related response. RT and N2 effects were present in averages and deconvolution output estimated from the raw data (data not shown), and remained significant in the residual activity after removal of confounding effects, that is, the error-preceding activity cannot be solely accounted for by skewed averaging of compatible/ incompatible trials or stimulus repetition priming.

What then is the mechanism behind error precursors? Predictive information conveyed by the stimulus history triggers neural and consequently behavioral adaptation. Local sequences with increased occurrence of a particular stimulus reduce the surprise 

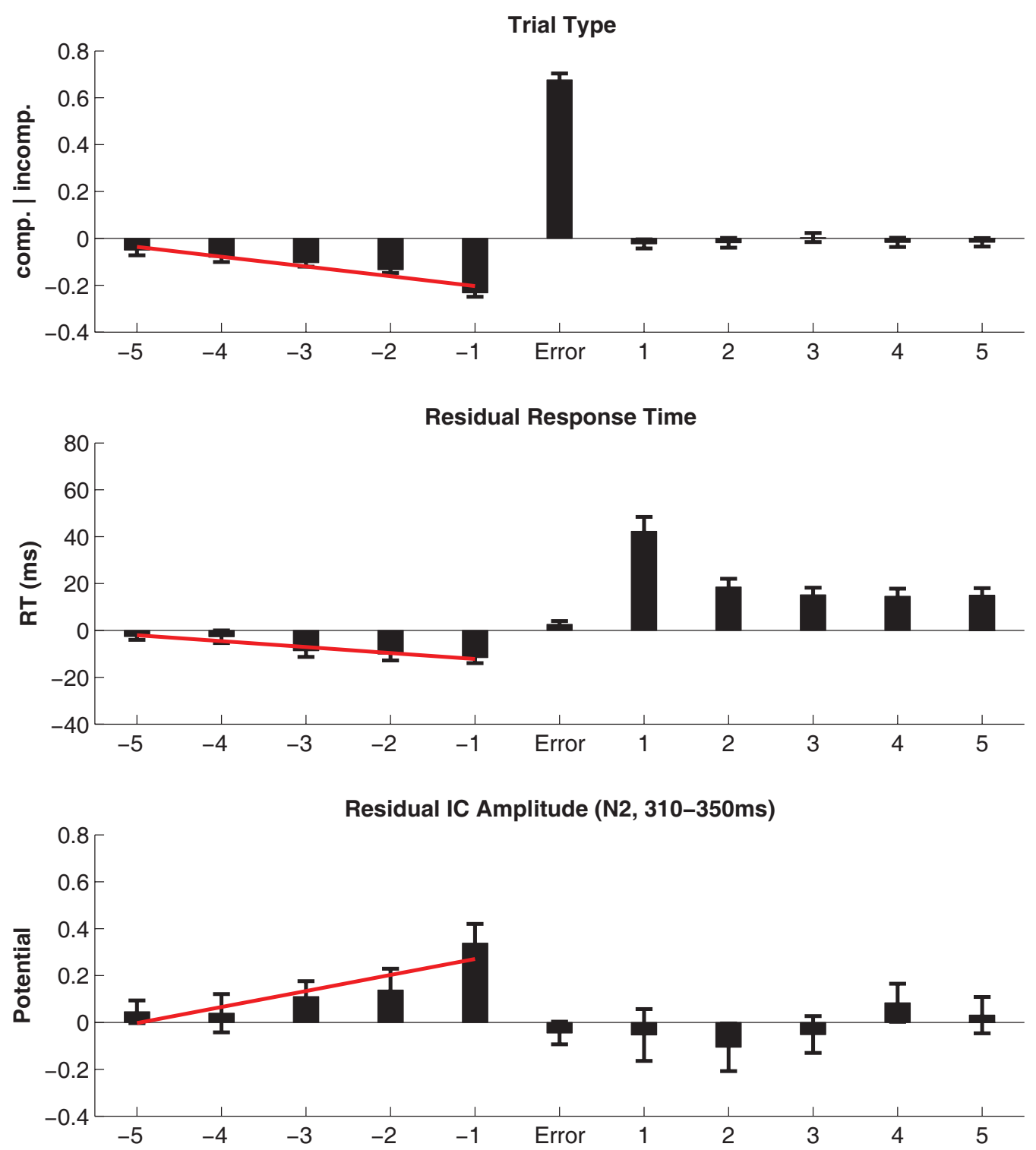

FIGURE 4 |Top panel: Most errors occurred to incompatible trials, and error-preceding trials showed an increased frequency of compatible trials. Following errors, the probability of compatible/incompatible outcomes is equal (as it is across the entire experiment). Middle panel: Residual RTs were on average $11 \mathrm{~ms}$ faster in trials immediately prior to errors and were speeding by $2.5 \mathrm{~ms}$ per trial from -5 to -1 . Post-error slowing of responses by $42 \mathrm{~ms}$ was observed and in subsequent trials sustained slowing on the order of $15 \mathrm{~ms}$ was present. Bottom panel: The residual amplitude at the N2 latency was less negative in trials prior to errors and a trend was present across the five error-preceding trials. Following errors, N2 estimates returned to baseline. Note that, in contradistinction to Figure 3 conflict-related N2 modulations as well as the error-related amplitude increase equivalent to the ERN/N2 have been removed by multiple linear regression. about the more frequent stimulus, reduce the local entropy in the stimulus sequence and thus increase the subjective predictability of upcoming stimuli (Huettel et al., 2002; Bestmann et al., 2008; Mars et al., 2008) and the possibility for "strategic modulation" (Gratton et al., 1992). As alluded to by the opening quote from the work of Squires and colleagues (Squires et al., 1976), generating predictions about upcoming stimuli/responses is a pervasive and automatic process that permits adaptive, optimized, fast and accurate responding to upcoming stimuli and has been shown on many levels of the cortical hierarchy (Squires et al., 1976; Llinas,
2001; Huettel et al., 2002; Eichele et al., 2005; Friston, 2005; Raichle, 2006; Bestmann et al., 2008). In the special case where low conflict compatible trials accumulate in the flanker task the mechanism becomes mal-adaptive, in the sense that RTs are speeding while conflict-monitoring-driven recruitment of effort by activity in the medial frontal wall is lowered concurrently, yielding a more error-prone trade-off between speed and accuracy (Forstmann et al., 2008; Ivanoff et al., 2008; van Veen et al., 2008). According to current theories of cognitive control the performance monitoring system provides signals for adaptive optimization of goal-directed 
behavior and signals the need for adjustments required in responses after errors and, more generally, whenever the action outcome is at risk (Botvinick et al., 2004; Ridderinkhof et al., 2004; Rushworth et al., 2007). Here, we show that the basic function of this system makes it equally susceptible to mal-adaptive over-optimization in the presence of random event series where local sequences convey low conflict and low uncertainty about future outcomes (Botvinick, 2007; Eichele et al., 2008). This is in line with the notion that the posterior medial frontal cortex plays a major role in effort-based cost-benefit valuations in humans (Croxson et al., 2009) and other animals (Schweimer et al., 2005; Rudebeck et al., 2006). In this way the gradual decline in N2 amplitude across trials preceding errors appears to reflect a reduction of attentional effort, which by itself is driven by increasing predictability.

Additionally, we should note that components in the N2 latency range serve as a match/mismatch detectors that are reduced by stimulus predictability and increased by surprise/prediction error (Eichele et al., 2005; Jongsma et al., 2006) in a similar way as the later P3 (Sutton et al., 1965; Mars et al., 2008), such that conflict-related and mismatch-related subcomponents, if represented together in the source analyzed here, might be reduced jointly (Forster et al., 2010; Folstein and Van Petten, 2008).

Considering the present results together with recent literature, it appears that apart from behavioral changes (Smith and Brewer, 1995; Gehring and Fencsik, 2001) and regional changes observed prior to errors in fMRI (Li et al., 2007; Eichele et al., 2008) there are a variety of features in the EEG signal that provide predictive information about upcoming RACC (Hajcak et al., 2005; Padilla et al., 2006; Cavanagh et al., 2009; Mazaheri et al., 2009; O’Connell et al., 2009). Future studies should clarify whether the diverse phenomenology describes aspects of the same underlying functional system or reflects multiple concurrent effects. For error prediction

\section{REFERENCES}

Allain, S., Carbonnell, L., Falkenstein, M., Burle, B., and Vidal, F. (2004). The modulation of the Ne-like wave on correct responses foreshadows errors. Neurosci. Lett. 372, 161-166.

Arieli, A., Sterkin, A., Grinvald, A., and Aertsen, A. (1996). Dynamics of ongoing activity: explanation of the large variability in evoked cortical responses. Science 273, 1868-1871.

Bell, A. J., and Sejnowski, T. J. (1995). An information-maximization approach to blind separation and blind deconvolution. Neural. Comput. 7, 1129-1159.

Bestmann, S., Harrison, L. M., Blankenburg, F., Mars, R. B., Haggard, P., Friston, K. J., and Rothwell, J. C. (2008). Influence of uncertainty and surprise on human corticospinal excitability during preparation for action. Curr. Biol. 18, 775-780.

Botvinick, M. M. (2007). Conflict monitoring and decision making: reconciling two perspectives on anterior cingulate function. Cogn. Affect. Behav. Neurosci. 7, 356-366.
Botvinick, M. M., Cohen, J. D., and Carter, C. S. (2004). Conflict monitoring and anterior cingulate cortex: an update. Trends Cogn. Sci. 8, 539-546.

Calhoun, V.D., Liu, J., and Adali, T. (2009). A review of group ICA for fMRI data and ICA for joint inference of imaging, genetic, and ERP data. Neuroimage 45:S163-S172.

Cavanagh, J. F., Cohen, M. X., and Allen, J. J. (2009). Prelude to and resolution of an error: EEG phase synchrony reveals cognitive control dynamics during action monitoring. J. Neurosci. 29, 98-105.

Croxson, P. L., Walton, M. E., O’Reilly, J. X., Behrens, T.E., and Rushworth, M.F. (2009). Effort-based cost-benefit valuation and the human brain. J. Neurosci. 29, 4531-4541.

Debener, S., Ullsperger, M., Siegel, M., and Engel, A. K. (2006). Single-trial EEG-fMRI reveals the dynamics of cognitive function. Trends $\operatorname{Cog} n$. Sci.10, 558-563.

Debener, S., Ullsperger, M., Siegel, M., Fiehler, K., von Cramon, D. Y., and Engel, A. K. (2005).

in real-world situations, it would be desirable to understand the effects of performance (mal-) adaptation on event-related and induced activities as well as connectivity to have multiple redundant features available that can be employed for classification.

In summary, we note that a combination of data mining and modeling on a trial-by-trial level helps to better understand the dynamics of the cognitive process under investigation and we hope this approach to analysis and inference becomes the rule rather than the exception in cognitive neuroscience and neuroimaging (Makeig et al., 2004; Debener et al., 2006; Onton et al., 2006). In this particular case we show that one antecedent of errors is indeed generated by the branch of the cognitive control system that is in charge of monitoring them.

\section{ACKNOWLEDGMENTS}

This paper provides a detail report of some of the data presented by the authors at the Opinions and Discussions on Cognitive Neuroscience symposium held in Amsterdam (10/26-10/27 2009). The work was supported by a Nevronor grant from the Norwegian Research council (189661) to Tom Eichele. Tom Eichele and Markus Ullsperger conceived the experiments; Heike Eichele, Hilde T Juvodden and undergraduate RA's collected the data; Heike Eichele and Tom Eichele analyzed the data; Heike Eichele, Hilde T Juvodden, Markus Ullsperger and Tom Eichele co-wrote the paper. We thank our undergraduate research assistants Aleksander $\mathrm{H}$. Erga, Eva Sunde, Katrine V. Granli, Christer Lunde Gjerstad, Ingrid Langøen Bull-Tornøe, Agnethe Smith Wiker, Pia E. Lyse, Branca Vojnovic, Camilla Lindgren and Marius Kalsås Worren for their help with data collection. We are indebted to Marco Steinhauser for helpful discussions. The data and analysis functions for trial sequence analysis are available from the corresponding author upon request.

Trial-by-trial coupling of concurrent electroencephalogram and functional magnetic resonance imaging identifies the dynamics of performance monitoring. J. Neurosci. 25, 11730-11737.

Delorme, A., and Makeig, S. (2004). EEGLAB: an open source toolbox for analysis of single-trial EEG dynamics including independent component analysis. J. Neurosci. Methods 134 9-21.

Eichele, T., Calhoun, V. D., and Debener, S. (2009). Mining EEG-fMRI using independent component analysis. Int. J. Psychophysiol. 73, 53-61.

Eichele, T., Debener, S., Calhoun, V. D., Specht, K., Engel, A. K., Hugdahl, K., von Cramon, D. Y., and Ullsperger, M. (2008). Prediction of human errors by maladaptive changes in event-related brain networks. Proc. Natl. Acad. Sci. U.S.A. 105, 6173-6178.

Eichele, T., Specht, K., Moosmann, M., Jongsma, M. L., Quiroga, R. Q., Nordby, H., and Hugdahl, K. (2005). Assessing the spatiotemporal evolution of neuronal activation with single-trial event-related potentials and functional MRI. Proc. Natl. Acad. Sci. U.S.A. 102, 17798-17803.

Eriksen, B. A., and Eriksen, C. W. (1974). Effects of noise letters upon identification of a target letter in a nonsearch task. Percept. Psychophys. 16, 143-149.

Falkenstein, M., Hohnsbein, J., Hoormann, J., and Blanke, L. (1991). Effects of crossmodal divided attention on late ERP components. II. Error processing in choice reaction tasks. Electroencephalogr. Clin. Neurophysiol. 78, 447-455.

Falkenstein, M., Hoormann, J., Christ, S., and Hohnsbein, J. (2000). ERP components on reaction errors and their functional significance: a tutorial. Biol. Psychol. 51, 87-107.

Folstein, J. R., and Van Petten, C. (2008). Influence of cognitive control and mismatch on the N2 component of the ERP: a review. Psychophysiology 45, 152-170.

Forster, S. E., Carter, C. S., Cohen, J. D., and Cho, R. Y. (2010). Parametric manipulation of the conflict signal and control-state adaptation. J. Cogn. Neurosci. (inpress). 
Forstmann, B. U., Dutilh, G., Brown, S., Neumann, J., von Cramon, D. Y., Ridderinkhof,K.R., andWagenmakers, E. J. (2008). Striatum and pre-SMA facilitate decision-making under time pressure. Proc. Natl. Acad. Sci. U.S.A. 105, 17538-17542.

Friston, K. J. (2005). A theory of cortical responses. Philos. Trans. R Soc. Lond. B Biol. Sci. 360, 815-836.

Gehring, W. J., and Fencsik, D. E. (2001). Functions of the medial frontal cortex in the processing of conflict and errors. J. Neurosci. 21, 9430-9437.

Gehring, W. J., Goss, B., Coles, M. G. H., Meyer, D. E., and Donchin, E. (1993). A neural system for error-detection and compensation. Psychol. Sci. 4, 385-390.

Gentsch, A., Ullsperger, P., and Ullsperger, M. (2009). Dissociable medial frontal negativities from a common monitoring system for self- and externally caused failure of goal achievement. Neuroimage 47, 2023-2030.

Gratton, G., Coles, M. G., and Donchin, E. (1992). Optimizing the use of information: strategic control of activation of responses. J. Exp. Psychol. Gen. 121, 480-506.

Hajcak, G., Nieuwenhuis, S., Ridderinkhof, K. R., and Simons, R. F. (2005). Error-preceding brain activity: robustness, temporal dynamics, and boundary conditions. Biol. Psychol. 70, 67-78.

Huettel, S. A., Mack, P. B., and McCarthy, G. (2002). Perceiving patterns in random series: dynamic processing of sequence in prefrontal cortex. Nat. Neurosci. 5, 485-490.

Huettel, S. A., Song, A. W., and McCarthy, G. (2005). Decisions under uncertainty: probabilistic context influences activation of prefrontal and parietal cortices. J. Neurosci. 25, 3304-3311.

Ivanoff, J., Branning, P., and Marois, R. (2008). fMRI evidence for a dual process account of the speed-accuracy tradeoff in decision-making. PLoS ONE 3, e2635. doi:10.1371/journal. pone. 0002635 .

James, W. (1890). The Principles of Psychology. New York: Holt.

Jongsma, M. L., Eichele, T., Van Rijn, C. M., Coenen, A. M., Hugdahl, K., Nordby, H., and Quiroga, R.Q. (2006). Tracking pattern learning with singletrial event-related potentials. Clin. Neurophysiol. 117, 1957-1973.

Klein, T. A., Endrass, T., Kathmann, N., Neumann, J., von Cramon, D. Y., and Ullsperger,M.(2007). Neural correlates of error awareness. Neuroimage 34, 1774-1781.
Kopp, B., Rist, F., and Mattler, U. (1996). N200 in the flanker task as a neurobehavioral tool for investigating executive control. Psychophysiology 33, 282-294.

Li, C. S., Yan, P., Bergquist, K. L., and Sinha, R. (2007). Greater activation of the "default" brain regions predicts stop signal errors. Neuroimage 38, 640-648.

Llinas, R. R. (2001). I of the Vortex: From Neurons to Self. Cambridge: The MIT Press.

Makeig, S., Debener, S., Onton, J., and Delorme, A. (2004). Mining eventrelated brain dynamics. Trends Cogn. Sci. 8, 204-210.

Mars, R. B., Debener, S., Gladwin, T. E., Harrison, L. M., Haggard, P., Rothwell, J.C., and Bestmann, S. (2008). Trial-bytrial fluctuations in the event-related electroencephalogram reflect dynamic changes in the degree of surprise. J. Neurosci. 28, 12539-12545.

Mayr, U., Awh, E., and Laurey, P. (2003). Conflict adaptation effects in the absence of executive control. Nat. Neurosci. 6, 450-452.

Mazaheri, A., and Jensen, O. (2008). Asymmetric amplitude modulations of brain oscillations generate slow evoked responses. J. Neurosci. 28, 7781-7787.

Mazaheri, A., Nieuwenhuis, I. L., van Dijk, H., and Jensen, O. (2009). Prestimulus alpha and mu activity predicts failure to inhibit motor responses. Hum. Brain Mapp. 30, 1791-1800.

Nagai, Y., Critchley, H. D., Featherstone, E., Fenwick, P. B., Trimble, M. R., and Dolan, R. J. (2004). Brain activity relating to the contingent negative variation: an $\mathrm{AMRI}$ investigation. Neuroimage 21, 1232-1241.

Notebaert, W., and Verguts, T. (2007). Dissociating conflict adaptation from feature integration: a multiple regression approach. J. Exp. Psychol. Hum. Percept. Perform. 33, 1256-1260.

O'Connell, R. G., Dockree, P. M., Robertson, I.H., Bellgrove, M.A., Foxe, J. J., and Kelly, S. P. (2009). Uncovering the neural signature of lapsing attention: electrophysiological signals predict errors up to $20 \mathrm{~s}$ before they occur. J. Neurosci. 29, 8604-8611.

Onton, J., Delorme, A., and Makeig, S. (2005). Frontal midline EEG dynamics during working memory. Neuroimage 27, 341-356.

Onton, J., Westerfield, M., Townsend, J., and Makeig, S. (2006). Imaging human EEG dynamics using independent component analysis. Neurosci. Biobehav. Rev. 30, 808-822.
Padilla, M. L., Wood, R. A., Hale, L. A. and Knight, R. T. (2006). Lapses in a prefrontal-extrastriate preparatory attention network predict mistakes. J. Cogn. Neurosci. 18, 1477-1487.

Raichle, M. E. (2006). Neuroscience. The brain's dark energy. Science 314, 1249-1250.

Raichle, M. E., MacLeod, A. M., Snyder, A. Z., Powers, W. J., Gusnard, D. A., and Shulman, G. L. (2001). A default mode of brain function. Proc. Natl. Acad. Sci. U.S.A. 98, 676-682.

Raichle, M. E., and Snyder, A. Z. (2007). A default mode of brain function: a brief history of an evolving idea. Neuroimage 37, 1083-1090; discussion 1097-1089.

Ridderinkhof, K. R., Nieuwenhuis, S., and Bashore, T. R. (2003). Errors are foreshadowed in brain potentials associated with action monitoring in cingulate cortex in humans. Neurosci. Lett. 348, 1-4.

Ridderinkhof, K. R., Ullsperger, M. Crone, E. A., and Nieuwenhuis, S. (2004). The role of the medial frontal cortex in cognitive control. Science 306, 443-447.

Roger, C., Benar, C. G., Vidal, F., Hasbroucq, T., and Burle, B. (2010). Rostral Cingulate Zone and correct response monitoring: ICA and source localization evidences for the unicity of correct- and error-negativities. NeuroImage 51, 391-403.

Rudebeck, P. H., Walton, M. E., Smyth, A N., Bannerman, D. M., and Rushworth, M.F. (2006). Separate neural pathways process different decision costs. Nat. Neurosci. 9, 1161-1168.

Rushworth, M. F., Buckley, M. J., Behrens, T. E., Walton, M. E., and Bannerman, D. M. (2007). Functional organization of the medial frontal cortex. Curr. Opin. Neurobiol. 17, 220-227.

Scheeringa, R., Bastiaansen, M. C., Petersson, K. M., Oostenveld, R., Norris, D. G., and Hagoort, P. (2008). Frontal theta EEG activity correlates negatively with the default mode network in resting state. Int. J. Psychophysiol. 67, 242-251.

Schweimer, J., Saft, S., and Hauber, W. (2005). Involvement of catecholamine neurotransmission in the rat anterior cingulate in effort-related decision making. Behav. Neurosci. 119, 1687-1692.

Smith, G. A., and Brewer, N. (1995). Slowness and age: speed-accuracy mechanisms. Psychol. Aging 10, 238-247.

Squires, K. C., Wickens, C., Squires, N. K., and Donchin, E. (1976). The effect of stimulus sequence on the waveform of the cortical event-related potential. Science 193, 1142-1146.

Stone,J.V.(2004). Independent Component Analysis: A Tutorial Introduction. Cambridge, MA: MIT press.

Sutton, S., Braren, M., Zubin, J., and John, E. R. (1965). Evoked-potential correlates of stimulus uncertainty. Science 150, 1187-1188.

Ullsperger,M.,Bylsma,L.M., and Botvinick, M. M. (2005). The conflict adaptation effect: it's not just priming. Cogn. Affect. Behav. Neurosci. 5, 467-472.

Ullsperger, M., and von Cramon, D. Y. (2004). Neuroimaging of performance monitoring: error detection and beyond. Cortex 40, 593-604.

van Veen, V., Krug, M. K., and Carter, C. S. (2008). The neural and computational basis of controlled speed-accuracy tradeoff during task performance. J. Cogn. Neurosci. 20, 1952-1965.

Viola, F. C., Thorne, J., Edmonds, B., Schneider, T., Eichele, T., and Debener, S. (2009). Semi-automatic identification of independent components representing EEG artifact. Clin. Neurophysiol. 120, 868-877.

Yeung, N., Botvinick, M. M., and Cohen, J. D. (2004). The neural basis of error detection: conflict monitoring and the error-related negativity. Psychol. Rev. 111, 931-959.

Yu, A. J., Dayan, P., and Cohen, J. D. (2009). Dynamics of attentional selection under conflict: toward a rational Bayesian account. J. Exp. Psychol. Hum. Percept. Perform. 35, 700-717.

Conflict of Interest Statement: The authors declare that the research was conducted in the absence of any commercial or financial relationships that could be construed as a potential conflict of interest.

Received: 18 May 2010; paperpending published: 02 June 2010; accepted: 21 July 2010; published online: 10 August 2010.

Citation: Eichele H, Juvodden HT, Ullsperger $M$ and Eichele $T$ (2010) Mal-adaptation of event-related EEG responses preceding performance errors. Front. Hum. Neurosci. 4:65. doi: 10.3389/ fnhum.2010.00065

Copyright (c) 2010 Eichele, Juvodden, Ullsperger and Eichele. This is an openaccess article subject to an exclusive license agreement between the authors and the Frontiers Research Foundation, which permits unrestricted use, distribution, and reproduction in any medium, provided the original authors and source are credited. 